\section{VEGF, VEGF165b and EG-VEGF expression is specifically related with hormone profile in pituitary adenomas}

\author{
Ana Silvia Corlan, ${ }^{1}$ \\ Anca Maria Cîmpean, ${ }^{1,2}$ Eugen Melnic, ${ }^{3}$ \\ Marius Raica, ${ }^{1,2}$ Simona Sarb ${ }^{1,2}$ \\ ${ }^{1}$ Department of Microscopic \\ Morphology/Histology, Victor Babeș \\ University of Medicine and Pharmacy, \\ Timișoara, Romania \\ ${ }^{2}$ Angiogenesis Research Center, Victor \\ Babeș University of Medicine and \\ Pharmacy, Timișoara, Romania \\ ${ }^{3}$ Department of Pathology, Nicolae \\ Testemiţanu State University of \\ Medicine and Pharmacy, Chișinău, \\ Moldova
}

\begin{abstract}
Vascular endothelial growth factor (VEGF), its inhibitory splice variant, VEGF $165 b$ and Endocrine Gland derived VEGF (EG-VEGF) have a controversial role in pituitary gland. We aim to study VEGF, VEGF $165 b$ and EG-VEGF expression in pituitary adenomas. A significant correlation was found between growth hormone $(\mathrm{GH})$ and VEGF secretion $(\mathrm{P}=0.024)$. For prolactinomas, VEGF and prolactin expression, had a P-value of 0.02 for Kendall coefficient and a P-value of 0.043 for the Spearman coefficient. VEGFmRNA amplification was detected in both tumor cells and folliculostellate cells. VEGF $165 b$ was positive in $16.66 \%$ of pituitary adenomas. EG-VEGF was significantly correlated with prolactin $(\mathrm{P}=0.025)$ and luteinizing hormone $(\mathrm{P}=0.028)$. Our data strongly support VEGF, VEGF $165 b$ and EG-VEGF as important players of pituitary adenomas tumorigenesis. Particular hormonal milieu heterogeneity, special vascular network with an unusual reactivity to tumor growth correlated with variability of VEGF, VEGF $165 b$ and EG-VEGF secretion may stratify pituitary adenomas in several molecular groups with a direct impact on therapy and prognosis.
\end{abstract}

\section{Introduction}

Vascular endothelial growth factor (VEGF) was first isolated from bovine pituitary as a vascular permeability factor and it plays an essential role in tumor angiogenesis by stimulating the proliferation and migration of endothelial cells, neovessels branching; all these properties have been studied in pituitary adenomas. ${ }^{1}$ Also, some data suggested that VEGF could increase cell survival by inducing anti-apoptotic protein bcl-2 expression in pituitary adenomas; the angiogenic activity of VEGF seems to be partially mediated by protecting the endothelial cells from apoptosis. ${ }^{2}$

Few papers reported correlations between hormone profile of pituitary adenomas and VEGF overexpression; most of these papers used immunohistochemistry for this purpose. ${ }^{3,4}$ Our study aims to reevaluate the expression and distribution of VEGF in the pituitary adenomas depending on hormone profile and to find if this expression may be used as a predictive factor for therapy and prognosis of pituitary tumors, especially of those with invasive and aggressive phenotype.

Despite of VEGF overexpression, several data reported a low microvessel density (MVD) in most of the pituitary tumors. These intriguing findings make us to think about the existence of an inhibitory factor in pituitary adenomas more or less related with VEGF. Only one paper recently published by Melnic, ${ }^{5}$ reported VEGF $165 b$ expression in pituitary adenomas. Thus, we consider that VEGF165b overexpression (the inhibitory variant of VEGF), in pituitary adenomas could explain, in part the lower MVD of pituitary adenomas compared with normal pituitary tissue.

A prokineticin family member EGVEGF (PROK1), has been described to selectively induce the proliferation and survival of endothelial cells in endocrine glands. ${ }^{6}$ EG-VEGF has been described in the ovary, testis, placenta and adrenal cortex. ${ }^{6,7}$ Raica et al. demonstrated for the first time a decrease of EG-VEGF expression in human pituitary adenomas, as compared to normal pituitary, and the authors concluded that the tumor cells may have an altered function in the release the hormones because of an altered tumor angiogenesis excepting LH-secreting pituitary adenomas which are not associated with a decreased expression of EG-VEGF, suggesting that LH could stimulate EG-VEGF secretion. ${ }^{8}$

\section{Materials and Methods}

\section{Patients and tumor samples}

Our study included 92 cases as follow: 10 normal pituitary glands obtained from autopsies and 82 cases of patients diagnosed with clinically and radiologically certified pituitary adenomas, including those prolactinomas which grew quickly and induced compression to adjacent structures.
Correspondence: Prof. Anca Maria Cîmpean, Department of Microscopic Morphology/ Histology, Angiogenesis Research Center, Victor Babeș University of Medicine and Pharmacy, Piata Eftimie Murgu 2, 00041 Timisoara, Romania.

Tel. +40.720060955

E-mail: ancacimpean1972@yahoo.com

Key words: VEGF; VEGF165b; EG-VEGF; pituitary adenomas; angiogenesis.

Conflict of interest: The authors declare that they have no conflict of interest.

Funding: The present work was supported by the Victor Babes University of Medicine and Pharmacy, Timișoara, Romania.

Received for publication: 6 January 2019.

Accepted for publication: 8 February 2019.

This work is licensed under a Creative Commons Attribution-NonCommercial 4.0 International License (CC BY-NC 4.0).

(C) Copyright A.S. Corlan et al., 2019

Licensee PAGEPress, Italy

European Journal of Histochemistry 2019; 63:3010 doi:10.4081/ejh.2019.3010

Tumor samples were collected by open surgery or by trans-sphenoidal approach. Informed consent was obtained from all patients regarding surgery approval and the use of their samples for research purpose. The local Research Ethics Committee approved the study protocol. All ethical issues of the present study overlapped to World Medical Association Declaration of Helsinki statements.

\section{Primary processing and routine histopathologic diagnosis}

Tissue samples were formalin fixed for 48-72 $\mathrm{h}$ and paraffin embedded. Three micrometer thick serial sections were obtained from each paraffin block and specimens were stained by using conventional hematoxylin and eosin method. We established the histopathological diagnosis of pituitary adenomas according to the WHO classification.

\section{Immunohistochemistry}

The complete hormonal profile included the assessment of 6 types of pituitary hormones: growth hormone $(\mathrm{GH})$, adrenocorticotrophic hormone (ACTH), thyroid stimulating hormone (TSH), folliculo-stimulating hormone (FSH), luteinizing hormone (LH) and prolactin (PRL) together with antibodies for VEGF, VEGF $165 b$ and EG-VEGF. All primary antibodies against pituitary hormones were kindly provided by Dako Cytomation (Carpinteria, CA, USA) 
being ready to use and thus, a dilution was not necessary. Positive immunostaining was considered as being positive if more than $10 \%$ of tumor cells were labelled. The immunolabelling index was graded as being 0 (negative), $1+(10-30 \%$ of cells), $2+(31-$ $50 \%$ of cells) or $3+$ (over $50 \%$ of cells). The tumors with high co-expression $\mathrm{GH} / \mathrm{PRL}$ immunoreactivity $(>50 \%$ of cells) were considered mammosomatotrophic adenomas. Regarding VEGFs antibodies, several sources were used. VEGF (mouse anti human monoclonal antibody, clone VG1, dilution $1: 25$ ) was provided by the same supplier as antibodies for pituitary hormones. We performed a dilution of 1:100 for EG-VEGF antibody (polyclonal, goat anti human, T16; Santa Cruz Biotechnology, Inc., Santa Cruz, CA, USA) and for VEGF165b (monoclonal mouse anti-human; Realitech, Germany) we chose a dilution of 1:25 as optimal for our purpose. We used as negative control pituitary tumor specimens where the primary antibody was omitted. Positive controls were represented by tubular structures of the kidney for VEGF, renal glomerular capillaries for VEGF $165 b$ and adrenal for EG-VEGF.

Briefly, immunohistochemistry included 30 min incubation with the primary antibodies, followed by the Novolink Max Polymer System (Leica Microsystems) as secondary antibody and 3,3'-diaminobenzidine as chromogen. We applied a score to quantify the positive immunohistochemical reaction based upon the incidence of positive cells for the 3 investigated growth factors (VEGF, VEGF165b, EG-VEGF) in association with the intensity of the positive reaction as it is described in Table 1 .

Immunohistochemistry was validated by in situ hybridization method for RNA, $R N A s c o p e$, by evaluating ribonucleic acid through the visualization of a single molecule in individual cells on the specimens that were formalin fixed and paraffin embedded.

The protocol was similar to that described by Wang et al. ${ }^{9}$ Tissue sections were pretreated to permeabilize cells, double $\mathrm{Z}$ probes hybridize to target RNA molecules, after that the hybridization signals are amplified via sequential hybridization of amplifiers and label probes and finally, the RNA molecules were visualized as brown

Table 1. Evaluation score for growth factors immunoexpression.

\begin{tabular}{lcc} 
Parameter & Intensity & Incidence \\
Mild & +1 & $<10 \% ;+1$ \\
Moderate & +2 & $10-50 \% ;+2$ \\
\hline Intense & +3 & $>50 \% ;+3$ \\
\hline
\end{tabular}

signals using the chromogen 3,3'-diaminobenzidine. VEGF-mRNA amplification was classified in 5 degrees: 0 ( no staining or less than 1 point for every 10 cells, $\mathrm{x} 400$ magnification), 1(1-3 points/cell, $\mathrm{x} 400$ magnification), 2 (4-10 points/cell, very few clusters of points visible at $\times 400$ magnification), 3 ( $>10$ points/cell, $<10 \%$ positive cells with clusters of points visible at x400 magnification), 4 ( $>10$ points/cell, $>10 \%$ of positive cells with clusters of points visible at $\times 400$ magnification).

\section{Image acquisition, data processing and statistical analysis}

Microscopic evaluation was performed by using Nikon Eclipse E600 (Nikon Corporation, Tokyo, Japan). Images were captured and processed with LuciaNet (Nikon) software. Statistical analysis was performed based on our results by using SPSS software version 20. A P-value of 0.05 or less was considered statistically significant. We made a statistical analysis using Pearson, Spearman and Kendall correlation parameters applied for immunohistochemical expression of pituitary hormones and growth factors immunoexpression. We considered as strong significant correlation when all three correlation parameters had a P-value less than 0.05. When just Spearman and Kendall parameters had a P-value of 0.05 or less, we considered that a partial significant correlation (weak significant correlation) existed.

\section{Results}

The histopathologic evaluation of pituitary adenomas revealed that $60.63 \%$ out of 82 cases of pituitary adenomas were acidophilic, 9.85\% were basophilic, $4.92 \%$ were chromophobe and $24.6 \%$ were of mixed type. A percent of $47.35 \%$ of cases had a solid growth pattern, $7.04 \%$ a papillary pattern and the resting ones being of trabecular, diffuse or combined patterns. According to immunohistochemical evaluation of pituitary hormones, $37.72 \%$ were immunopositive for $\mathrm{GH}, 18.86 \%$ had positive reaction for PRL, $8.61 \%$ were mixed GH and PRL expressing adenomas, $1.7 \%$ were positive for $\mathrm{ACTH}, 0.65 \%$ for $\mathrm{TSH}$, $9.84 \%$ for $\mathrm{FSH}-\mathrm{LH}, 7.46 \%$ were plurihormonal and $15.16 \%$ did not express any pituitary hormones being considered as nonsecreting adenomas.

The VEGF immunoexpression in pituitary adenomas was found positive in $45 \%$ out of total cases. We observed 2 patterns of VEGF expression: a membrane pattern, with increased and homogeneous intensity through the entire circumference of the positive tumor cells and a cytoplasmic granular pattern. In some cases, we observed both patterns expressed in the same tumor cell or, in the case of mixed pituitary adenomas, in different types of tumor cells that form the adenoma. Pertaining the distribution area of the immunohistochemical expression of VEGF, we identified 2 types of expression: a diffuse and a focal expression. The diffuse

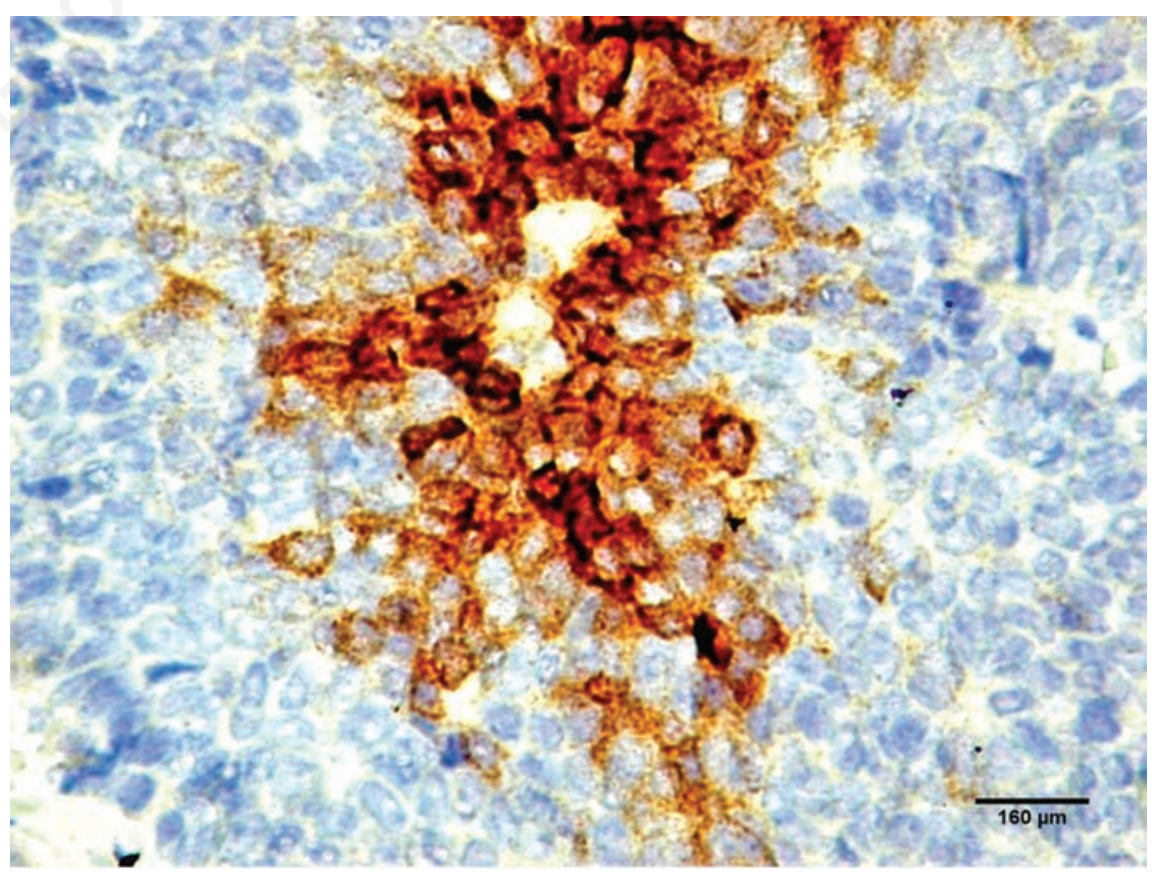

Figutr 1. Island of VEGF-positive cells in an ACTH-secreting pituitary adenoma. 
expression pattern was specific for $\mathrm{GH}$ and PRL secreting pituitary adenomas. In TSH, ACTH and LH secreting adenomas, we identified a focal expression pattern. The immunohistochemical reaction for VEGF was mild or absent in most of the nonsecreting pituitary adenomas. From all VEGF positive cases, $51.85 \%$ were pure acidophilic pituitary adenomas or mixed ones with an acidophilic component, 25\% were pituitary adenomas with chromophobe cells and $23.5 \%$ were of basophil type.

Based on hormone profile, we found differences regarding VEGF immunoexpression. GH secreting adenomas had a high VEGF expression, a significant correlation being found between GH and VEGF secretion having a P-value of 0.024 $(\mathrm{r}=0.014)$. For prolactinomas, a partial significant correlation has been found with VEGF expression, obtaining a P-value of 0.02 for the Kendall correlation coefficient and a P-value of $0.043 \quad(\mathrm{r}=0.425)$ for Spearman correlation coefficient.

Mixed pituitary adenomas with both GH and PRL secretion, had a moderate intensity of VEGF expression, quantified by +2 . The mixed pituitary adenomas, secreting both GH and TSH, had an intense positive reaction, quantified by +3 . In pituitary adenomas positive for ACTH and TSH, we observed an interesting pattern of VEGF expression: groups of cells that were positive for VEGF surrounded by negative cells for VEGF (Figure 1). Pseudo-vascular spaces lined by VEGF positive cells suggesting vasculogenic mimicry has been detected in prolactin secreting pituitary adenomas and never seen in other types of pituitary adenomas included in the study. VEGF immunoexpression needed confirmation by RNAscope method. By this method, we found VEGF-mRNA amplification in $88.24 \%$ out of pituitary adenomas previous- ly found positive for VEGF by immunohistochemistry. We obtained a heterogenous gene amplification pattern for VEGF in the pituitary adenomas, with scores from +1 to +4 . For $53.33 \%$ of adenomas we classified as having a +3 and +4 scores, sustaining a moderate and increased amplification pattern in more than half of the cases. VEGFmRNA amplification was correlated with growth pattern of the pituitary adenomas. The papillary growth pattern showed in all cases a gene amplification pattern quantified as +4 . Concerning the papillary pattern from acidophilic type of pituitary adenomas, VEGF-mRNA amplification was observed in all cells (Figure 2a).

We observed a high gene amplification pattern also in the solid growth pattern, but, here, we identified a majority of distinct punctiform nuclear signals (Figure 2 b,c) and, to a lesser extent clusters of amplification (Figure 2d). VEGF-mRNA amplifica-

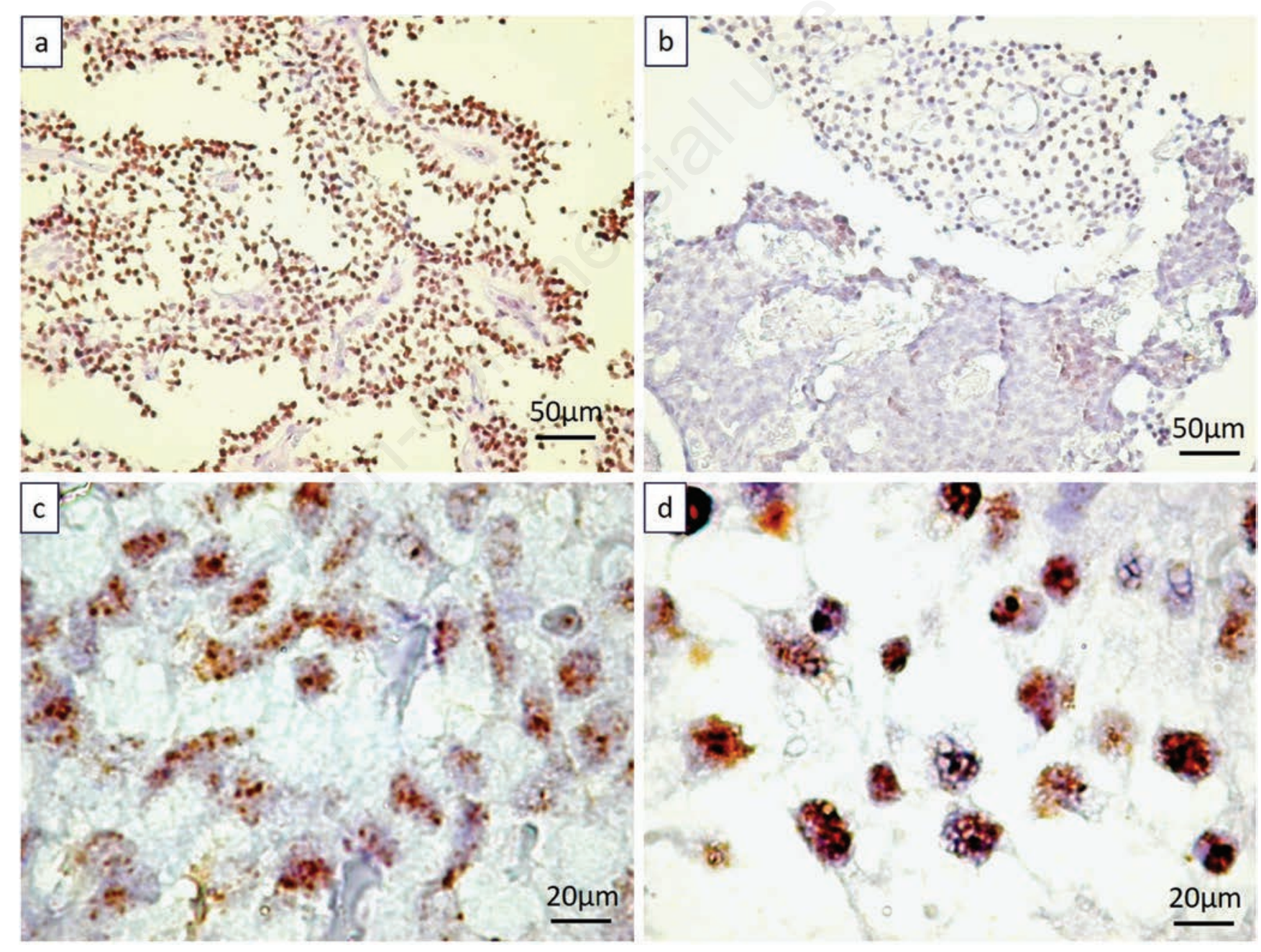

Figure 2. VEGF-mRNA amplification pattern in the papillary growth pattern with a score of +4 (a) and heterogenous amplification in a pituitary adenoma with solid and papillary component (b). Distinct nuclear signals for acidophilic cells of pituitary adenomas with a solid growth pattern (c), compared to the presence of clusters for acidophilic cells in papillary adenomas (d). 
tion was observed also in other cell types than tumor cells, mainly in endothelial cells from tumor blood vessels (Figure 3a), and neovessels with pillars inside suggesting an active intussusceptive mechanism of angiogenesis (Figure $3 \mathrm{~b}, \mathrm{c}$ ). VEGF-mRNA amplification was also detected in the cytoplasm and nucleus of folliculo-stellate cells (Figure 3d).

The VEGF $165 b$ expression in pituitary adenomas was assessed based of our previous observations regarding the decrease of vessels number in pituitary adenomas compared with normal pituitary gland. Immunohistochemistry revealed that $16.66 \%$ out of total cases were positive for VEGF $165 b$. For most of the pituitary adenomas, the immunostaining intensity was low, scored as $+1 ; 7$ pituitary adenomas had a moderate intensity, scored as +2 . All cases had granular cytoplasmic pattern of VEGF 165b immunoexpression few of them also showing an associated membrane pattern. All the adenomas positive for VEGF $165 b$ were acidophilic type or mixed, containing areas of acidophilic cells. In pituitary adenomas, negative for VEGF $165 b$ in tumor cells, we identified this growth factor in isolated cells, most probably macrophages or mast cells. We also identified folliculostellate cells positive for VEGF 165b. The VEGF $165 b$ expression pattern was granular cytoplasmic; $64 \%$ out of the pituitary adenomas expressed $E G$ $V E G F$. In $15 \%$ of the adenomas, the intensity was +1 , thus we considered them to be negative for EG-VEGF. Totally, $36 \%$ of the tumors were negative for EG-VEGF. In the normal pituitary gland, the expression of EG-VEGF was detected in most of acidophilic and chromophobe cells, but not in basophils ones; $32.6 \%$ of the pituitary adenomas, which were immunohistochemically positive for EG-VEGF, had a compact growth pattern; $28.26 \%$ had a papillary growth pattern, $19.56 \%$ had a mixed growth pattern.

Pertaining the adenomas with compact growth pattern, positive for EG-VEGF, $60 \%$ were GH secreting adenomas, with an intensity of reaction of +2 and +3 . Pituitary adenomas with a papillary growth pattern were all PRL positive adenomas by immunohistochemistry, and they also had positive reaction for EG-VEGF. We observed an increased intensity of the reaction in $70 \%$ of the cases; the reaction was homogenous, and EG-VEGF immunoexpression was present in the whole tumor area. We obtained a statistically significant correlation between EG-VEGF and PRL $(\mathrm{P}=0.025, \mathrm{r}=0.061)$. Also, for mixed pituitary adenomas, we established a significant correlation between EG-VEGF and LH $(\mathrm{P}=0.028, \mathrm{r}=0.8)$.

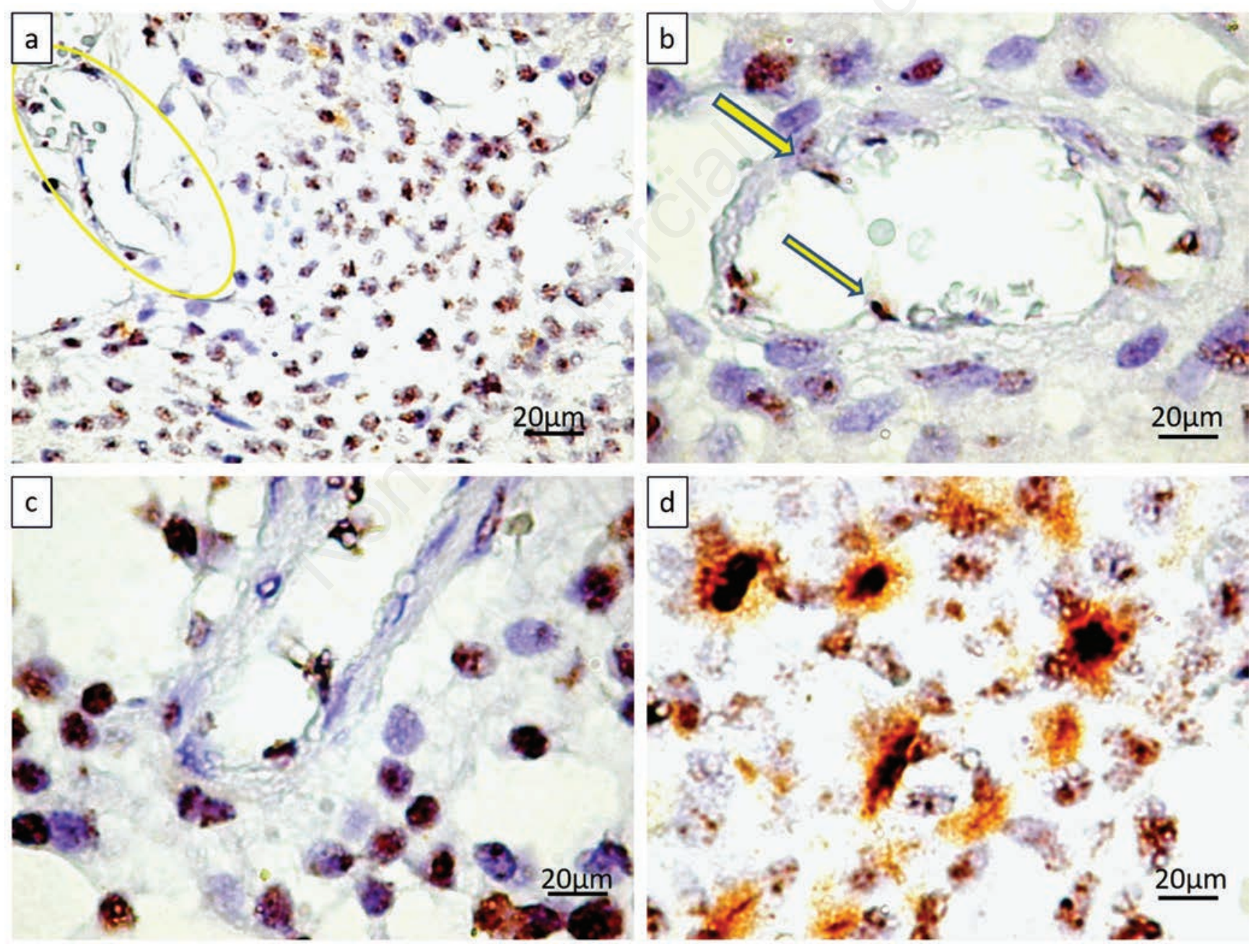

Figure 3. Other cell types that showed gene amplification pattern for VEGF-mRNA. Endothelial cells from peritumoral vessels (a) had an intense signal for VEGF-mRNA and in the vessels that were split by intravascular pillars, we observed endothelial cells with VEGFmRNA amplification at their emergent points (b, arrow) or in the structure of the pillar (c). The folliculostellate cells in between tumor cells, with an increased expression of VEGF-mRNA (d). 


\section{Discussion}

VEGF overexpression was reported in contrast with a decreased microvascular density in pituitary adenomas. There are some data reporting that the overexpression of VEGF is accompanied by an increased expression of endostatin in the majority of pituitary adenomas; endostatin is an endogenous inhibitor of angiogenesis and tumor growth and it is a C-terminal fragment of collagen type XVIII. Endostatin could explain the decreased MVD in pituitary adenomas. ${ }^{10}$ VEGF expression was detected in GH and PRL secreting pituitary adenomas; in these tumor types, we identified a significant correlation between VEGF expression and their hormonal profile. Our results are similar with data from the literature. We identified a particular expression pattern of VEGF in ACTH secreting adenomas, not reported before. The tumor cells positive for VEGF were grouped around vascular structures, other tumor cells being negative. A factor that could explain this insular growth pattern would be the increased proliferation rate of these tumor cells. This increased proliferation rate generated areas of cellular hypoxia which activate VEGF transcription. The identification of blood vessels in the tumor islands that express VEGF sustains a role for this growth factor in the tumorigenesis of ACTH and TSH secreting adenomas, but also in the stimulation of angiogenesis in these tumors. The tumor areas positive for VEGF and increased proliferation rate could generate tumors with invasive behavior - a pattern which was observed in different papers describing ACTH secreting adenomas. ${ }^{11}$ Our results indicate that the increased expression rate of VEGF characterizes the solid and papillary growth pattern of pituitary adenomas. Immunohistochemistry identified similar intensity of VEGF expression in these tumors. But, using RNAscope, we identified difference of gene amplification patterns: distinct nuclear signals in the solid growth pattern of pituitary adenomas and clusters of amplification in tumors with a papillary growth pattern. Till now, there were no data about the quantitative analysis of VEGF $\mathrm{m} R N A$ expression, using the RNAscope technique, in human pituitary adenomas.

The role of the folliculostellate cells in the pathogenesis of pituitary adenomas is explained through the synthesis and secretion of VEGF, a growth factor acting as mitogen for the tumor cells of pituitary adenomas. ${ }^{12}$ In our study we obtained similar results pertaining the expression of VEGF, at the protein and mRNA level. We observed a high protein level of VEGF in folliculostellate cells certified by VEGF
mRNA amplification. There were only two reports in the literature about VEGF expression in pituitary folliculostellate cells: one performed on sheep pituitary gland ${ }^{13}$ and another one on bovine pituitary tissue. ${ }^{14}$ Our study highlighted VEGF overexpression in pituitary folliculostellate cells of human origin.

VEGF $165 b$, the inhibitory splice variant of VEGF was downregulated in pituitary adenomas. The increased expression of VEGF $165 b$ correlated with VEGF overexpression only for $18 \%$ of the pituitary adenomas and this small percent of cases are not enough to explain the lack or decreased number of neovessels in tumor areas.

The low number of pituitary adenomas overexpressed VEGF165b had a particular pattern of its expression: the tumor cells with a positive immunohistochemical reaction for VEGF165b were grouped around mature blood vessels. This distribution pattern could explain the VEGF165b inhibitory effect on proliferation and migration of endothelial cells, with an inhibitory role in angiogenesis, a potential mechanism explaining the decreased microvascular density in pituitary adenomas. But the small proportion of pituitary adenomas, that overexpress VEGF165b implies the existence of other inhibitory mechanisms of tumor angiogenesis in pituitary adenomas. Endostatin (an endogenous inhibitor of angiogenesis previously reported to be identified in significant quantities in these pituitary adenomas $)^{10}$ may be one of these mechanisms. In pituitary adenomas, where we did not identify the expression of VEGF165b in tumor cells by using immunohistochemistry, we identified this growth factor in stromal cells having a morphology of mast cells and macrophages. This observation could represent another mechanism of tumor angiogenesis inhibition in pituitary adenomas with negative tumor cells for VEGF 165b. In these adenomas, an accumulation of VEGF $165 b$ positive macrophages or mast cells, could represent an important source of VEGF $165 b$.

EG-VEGF its less studied in pituitary adenomas and normal pituitary gland. ${ }^{3}$ In the normal pituitary gland, we identified the expression of EG-VEGF in acidophilic and chromophobe cells, but not in basophil cells. Previous papers reported an EGVEGF overexpression in several malignant tumors, arising from the gastrointestinal tract, ${ }^{15}$ pancreas, ${ }^{16}$ ovaries, ${ }^{7}$ or colon. ${ }^{17}$ In these tumors, EG-VEGF has an important role in tumor angiogenesis, controlling the tumor invasion and metastasis, but also the rate of proliferation of malignant cells. ${ }^{18,19}$ We also identified an overexpression of EGVEGF in more than $50 \%$ of pituitary adeno- mas. EG-VEGF may stimulate the phosphorylation of the receptor for EGF and its activation, thus having an antiapoptotic role and stimulating the survival of tumor cells. ${ }^{15}$ In pituitary adenomas with papillary growth pattern, we identified a correlation between the expression of EG-VEGF and PRL. It is well known that EG-VEGF and PRL have mitogenic role upon cells expressing their receptors. In our group of pituitary adenomas, where we had several prolactinomas, we identified an overexpression of EG-VEGF; in these tumors, we previously observed an increased Ki67 proliferation index, which was significantly correlated with the expression of EG-VEGF in tumor cells. In conclusion, there is a synergy of the mitogenic action of both EGVEGF and PRL, which stimulate the proliferation of tumor cells followed by an increase of tumor volume. In normal pituitary cells, the basophilic cells did not express EG-VEGF but the basophilic pituitary adenomas had a positive immunohistochemical reaction for EG-VEGF. These basophilic pituitary adenomas, with EGVEGF expression were of papillary type. There are papers that imply that EG-VEGF has a role in determining the tumor papillary growth pattern in other endocrine glands, like thyroid. ${ }^{20}$ In our group of tumors, there was pointed out a correlation, statistically significant, between increased EG-VEGF and LH, similar to the observations already published in the literature. ${ }^{21}$

We identified in our study EG-VEGF expression in folliculostellate cells. The papers published until now, do not prove the synthesis of EG-VEGF in folliculostellate of pituitary adenomas; there are no information that could confirm an action of EGVEGF in folliculostellate cells from the normal or tumor pituitary gland.

Our data strongly support VEGF, VEGF $165 b$ and EG-VEGF as important players of pituitary adenomas tumorigenesis. Particular microenvironment given by hormones heterogeneity, special vascular network with an unusual reactivity to tumor growth correlated with variability of VEGF, VEGF 165b and EG-VEGF secretion may stratify pituitary adenomas in several molecular groups with a direct impact on therapy and prognosis.

\section{References}

1.Turner HE, Nagy Z, Gatter KC, Esiri MM, Harris AL. Angiogenesis in PArelationship to endocrine function, treatment and outcome. J Endocrinol 2000;2:475-81.

2. Nor JE, Christensen J, Mooney DJ, Polverini PJ. Vascular endothelial growth 
factor (VEGF)-mediated angiogenesis is associated with enhanced endothelial cell survival and induction of Bcl-2 expression. Am J Pathol 1999;154:375-84.

3. Viacava P, Gasperi M, Acerbi G, Manetti L, Cecconi E, Bonadio AG, et al. MVD and VEGF expression in normal pituitary tissue and pituitary adenomas. J Endocrinol Invest 2003;26:23-8.

4. Lloyd RV, Scheithauer BW, Kuroki T, Vidal S, Kovacs K, Stefaneanu L. VEGF expression in human pituitary adenomas and carcinomas. Endocr Pathol 1999;10: 229-35.

5. Melnic E. The role of VEGF 165b in pituitary adenomas pathogenesis. Res Clin Med, 2017;1:11-2

6. Miyamoto S, Yagi H, Yotsumoto F, Kawarabayashi T, Mekada E. Heparinbinding epidermal growth factor-like growth factor as a novel targeting molecule for cancer therapy. Cancer Sci 2006; 5:341-47.

7. Ngan ES, Lee KY, Yeung WS, Ngan HY, $\mathrm{Ng} \mathrm{EH}$, Ho PC. Endocrine gland derived vascular endothelial growth factor is expressed in human peri-implantation endometrium, but not in endometrial carcinoma. Endocrinology 2006;147:88-95.

8. Raica M, Coculescu M, Cimpean AM, Ribatti D. EG-VEGF is downregulated in human pituitary adenomas. Anticancer Res 2010;30:3981-86.

9. Wang F, Flanagan J, Su N, Wang LC, Bui $\mathrm{S}$, Nielson A, et al. RNAscope a novel in situ RNA analysis platform for formalinfixed, paraffin- embedded tissues. J Mol Diagn 2012;14:22-9.
10. Lee KM, Park SH, Park KS, Hwang JH, Hwang SK. Analysis of circulating endostatin and vascular endothelial growth factor in patients with pituitary adenomas treated by stereotactic radiosurgery: A preliminary study. Brain Tum Res Treat 2015;3:89-94.

11. Rotondo F, Cusimano M, Scheithauer BW, Coire C, Horvath E, Kovacs K Atypical, invasive, recurring Crooke cell adenoma of the pituitary. Hormones 2012;11:94-100.

12. Turner HE, Nagy Z, Gatter KC, Esiri MM, Harris AL, Wass JA. Angiogenesis in pituitary adenomas and the normal pituitary gland. J Clin Endocrinol Metab 2000;85:1159-62.

13. Jabbour HN, Boddy SC, Lincoln GA. Pattern and localisation of expression of vascular endothelial growth factor and its receptor flt-1 in the ovine pituitary gland: expression is independent of hypothalamic control. Mol Cell Endocrinol 1997;134:91-100.

14. Gospodarowicz D, Lau K. Pituitary follicular cells secrete both vascular endothelial growth factor and follistatin. Biochem Biophys Res Commun 1989; 165:292-8.

15. Corlan AS, Cîmpean AM, Jitariu AA, Melnic E, Raica M. Endocrine glandderived vascular endothelial growth factor/prokineticin-1 in cancer development and tumor angiogenesis. Int $\mathrm{J}$ Endocrinol 2017;2017:3232905.

16. Vilchis MA, Chavez B, Chan C, RoblesDiaz G, Diaz Sanchez V. Expression and localization of EG-VEGF in human pan- creas and pancreatic adenocarcinoma. J Steroid Biochem Mol Biol 2007;107:3741.

17. Goi T, Fujioka M, Satoh Y, Tabata S, Koneri K, Nagano H, et al. Angiogenesis and tumor proliferation/metastasis in human colorectal cancer cell line SW620 transfected with EG-VEGF, as a new angiogenic factor. Cancer Res 2004;64: 1906-10.

18. Ngan ESW, Sit FYL, Lee KL, Miao X, Yuan Z, Wang W, et al. Implications of EG-VEGF/prokineticin-1 signaling in human neuroblastoma progression. Clin Cancer Res 2007;13:868-75.

19. Samson M, Peale FV, Frantz G, RiouxLeclercq N, Rajpert-De Meyts E, Ferrara N. Human EG-VEGF: expression early in development and in Leydig cells tumors suggests roles in normal and pathological testis angiogenesis. J Clin Endocrinol Metab 2004;89:4078-88.

20. Pasquali D, Santoro A, Bufo P, Conzo G, Deery WJ, Renzullo A, et al. Upregulation of endocrine gland-derived vascular endothelial growth factor in papillary thyroid cancers displaying infiltrative patterns, lymph node metastases, and BRAF mutation. Thyroid 2011;21: 391-9.

21. Hoffmann P, Saoudi Y, Benharouga M, Graham CH, Schaal JP, Feige JJ, et al. Role of EG-VEGF in human placentation: physiological and pathological implications. J Cell Mol Med 2009;13: 2224-35. 